\title{
TUBERCULOSIS INFANTIL
}

Por el Dr. JULIO ESPINOZA s.

Instituto de Anatomia-Patológica del Hospitisl de Niños "M. Arriarán".

Nos ha parecido de interés realizar un estudio anátomo-patológico de la tuberculosis del niño, aprovechando nuestro abundante material necrópsico, por la importancia que reviste esta enfermedad en nuestro medio, dada su alta frecuencia. Este trabajo hemos tratado de realizarlo, abordando algunos de los múltiples aspectos con que la enfermedarl tuberculosa se presenta en el niño y en cada caso hemos procurado comentar y comparar nuestros resultadós con la experiencia de autores nacionales y extraijeros, Toda la patología que contiene el trabajo, se refiere primordialmente a la infección primaria tuberculosa y a las consecuencias derivadas de ésta, cuanảo en vez de involucionar, sigue tna marcha progresiva, dando lugar a la serie de manifestaciones patológicas, pulmonares o extrapulmonares, localizadas 0 generalizadas, etc., que suelen poner en grave peligro la vida del niño. En consecuencia, sólo por excepción, nos reforiremos a algunos casos con tuberculosis $d_{0}$ reinfección, pero sin siquiera intentar un estudio sistemático de ellos.

Otra razón que nos mopió a la realización de este trabajo y a hacerlo desde un punto de vista panorámico, fué el uso cada dia más generalizado en nuestro medio, de los antibióticos, especialmente de la estreptomicina, los cuales, según la experiencia gue dejamos transcrita, altora y transforma los cuadros patológicos, haciondo entonces muy difícil o imposible, seguir en el cadáver el desarrollo de la enfermedad tuberculosa en sus diversas fases, conocimiento que, a nuestro juicio, os de sumo interés para desentrañar las múltiples incógnitas que existen en la patogenia de esta enfer- medad y que tan enorme trascendencia tiene en el terteno clínico. La generalización on el uso de estos medicamentos, que día a día se multiplican, hará que en el futuro sean de difícil o rara observación los cuadros anatómicos más o menos clásicos a que da lugar la primoinfección tuberculosa y de aquellos otros: que son secundarios a ella, obligándonos a recurrir, para la comprensión y estudio de la patogenia de estos procosos, a trabajos morfológicos anteriores a la presente etapa que vivimos y que por desgracia son, entre nosotros, relativamente escasos. Sin pretender agotar los temas que aqui abordamos, creemos que por el número de casos y el análisis cuidadoso que de ellos hemos tealizado, puede servir en el futuro como exporioncia fundamental de nuestra patología tuberculosa.

Nuestro material está constituído por 3,009 autopsias, practicadas en el Instituto de Anatomía Patológica del Hospital de Niños "Manuel Arriarán", durante los años 1943 a 1949.

El desarrollo del presente trabajo está sujeto al siguiente orden:

Capitulo I: a). Morbilidad y mortalidad, y b) Causa de muerte de los fallecidos por en.fermedad no tuberculosa.

Capítulo II: a) Complejo primario; b) Chancro primario pulmonar; c) Ausencia de una parte del complejo primario; d) Infección primaría extrapuImonar, y e) Ausencia de complejo primario.

Capítulo IH: Estado físico del chancro pulmonar.

Capítulo IV: Perforación de órganos torácicos por chancros, cavernas y ganglios tuberculosos.

Capitulo V: Tuberculosis miliar generalizada. 
Capitulo VI: Leptomenjmgitis tuberculosa.

Capitulo VII: Tụberculosis orgánica.

Capítulo VII: Infección linfáttca retrógrada en la tuberculosis ganglionar.

Conclusiones.

Resumen.

Blbllografía.

\section{I}

\section{a) Morbilided y mortalidad.}

De acuerdo con Terplan ${ }^{1}$, Aschoff ${ }^{2}$ y otros auteres, la incidencia real de la tuberculosis sólo puede obtenerse mediante prolija investigación del material necrópsico. Para estos efectos, estos autores utilizan no sólo la disección anatómica sino el estudio radiográfico de los órganos torácicos, lo cual permite el hallazgo de pequeños complejos pulmonares o ganglionares, que pueden fácilmente pasar desapercibidos a la simple inspección macroscópica. Por desgracia, nosotros sólo hemos podido contar con los procedimientos corrientes de disección.

Los hallazgos de lesiones tuberculosas, junto con el porcentaje correspondiente de positividad, pueden verse en el cuadro $N^{\circ}$ I. El caso más joven con taberculosis fué el de un niño de 1 mes 25 dias, lo cual no es excepcional, ya que entre esta edad y los 2 meses habir un total de 5 casos. Para hacer más elocuente los porcentajes de positividad,

\section{Cuadro $\mathrm{N}^{4} 1$}

Morbilidad de la tuberculosis en diferentes edades.

\begin{tabular}{rrrr}
\hline Edad & $\begin{array}{c}\text { N }^{9} \\
\text { autop. }\end{array}$ & $\begin{array}{l}\text { Casas } \\
\text { posit. }\end{array}$ & $\begin{array}{c}\% \\
\text { posit. }\end{array}$ \\
\hline I mes a 12 meses & 1881 & 94 & $\mathbf{4 . 9 9}$ \\
13 meses a 24 meses & 487 & 99 & 20.32 \\
2 años a 4 años & 252 & 99 & 39.28 \\
4 años a 6 años & 110 & 52 & 47.27 \\
6 años a 8 años & 10.0 & 45 & $45 .-$ \\
8 años a 10 años & 83 & 34 & 40.96 \\
10 años a 12 años & 79 & 47 & 59.49 \\
12 años a 14 años & 15 & 6 & $40 .-$ \\
14 años a 16 años & 2 & 2 & $100 .-$
\end{tabular}

comparemos nuestros resultados con los de Terplan (Buffalo, N. Y.) (cuadro $\mathrm{N}^{\circ}$ 2). Como es fácil observar, las diferencias en los porcentajes son sencillamente enormes, sobrepasando los nuestros 2, 3 y 4 veces a los de Terplan. No nos corresponde analizar las causas de esta mayor morbilidad en nuestro país. Sólo nos interesa dejar constancia de las cifras para que sean estudiadas por otras personas u organismos competentes. Sólo queremos agregar, aunque sea redundancia, que el resultado de la morbilidad correspondiente a la edad sobre 14 años, ro puede considerarse real, ya que el número de casos es estadísticamente sin inportancia

Si consideramos que el número total da casos con lesiones tuberculosas, alcan-

Cuadro $\mathrm{N}^{*} 2$

Comparación de los resultados de Terplan con los nuestros.

\begin{tabular}{|c|c|c|c|c|c|c|}
\hline Edad & $\begin{array}{l}\mathrm{N}^{9} \\
\text { autop. }\end{array}$ & $\begin{array}{c}\text { Te r p } 1 \\
\text { Casos } \\
\text { posit. }\end{array}$ & $\begin{array}{l}\text { Porcen- } \\
\text { taje }\end{array}$ & $\begin{array}{c}\mathbf{N}^{\circ} \\
\text { autop. }\end{array}$ & $\begin{array}{c}\text { Nosotr } \\
\text { Casos } \\
\text { posit. }\end{array}$ & $\%$ \\
\hline 5 semanas a 12 meses & 345 & 10 & 3.4 & 1881 & 94 & 4.99 \\
\hline 13 meses a 2 años & 97 & 10 & 9.7 & 487 & 99 & 20.32 \\
\hline 2 años a 6 años & 144 & 15 & 10.4 & 362 & 151 & 41.71 \\
\hline 6 años a 10 años & 40 & 7 & 17.5 & 183 & 79 & 43.18 \\
\hline 10 años a 14 años & 40 & 8 & 27.5 & 94 & 53 & 56.38 \\
\hline 14 años a 18 años & 32 & 7 & 21.8 & 2 & 2 & 100. \\
\hline 5 semanas a 6 años & 5896 & 35 & 5.9 & 2730 & 344 & 12.60 \\
\hline 6. años a 18 años & 112 & 22 & 19.4 & 279 & 134 & 48.02 \\
\hline
\end{tabular}


zan en nuestro material a 478 , la morbilidad general, desde el punto de vista anátomo-patológico, corresponde al $15.88 \%$, mientras que el de Terplan es de $8.16 \%$. De los 478 casos, murie-

\section{Cuadro $\mathrm{N}^{\circ} 3$}

Mortalidad de la tuberculosis en diferentes edades.

\begin{tabular}{ccc} 
No & $\begin{array}{c}\text { Ni de } \\
\text { autop. } \\
\text { muertos } \\
\text { por tbc. }\end{array}$ & $\begin{array}{c}\text { Porcen- } \\
\text { taje }\end{array}$ \\
\hline
\end{tabular}

1 mes a 12 meses 1881

13 meses a 24 meses 487

2 años a 4 años

4 años a $B$ años

6 años a 8 años

8 añas a 10 años

10 años a 12 ainos

12 años a 14 años

14 años a 16 años

Totales

\section{2}

110

100

83

78

15

2
3.88

16.63

30.95

34.54

30.-

30

32.50

45.56

40. -

100.-

\section{$3009 \quad 371(12.32 \%)$}

ron por tuberculosis 371 , lo que significa una mortalidad general del $12.32 \%$. Según los datos que Terplan da en su trabajo, la mortalidad en su material, sólo es del $5.01 \%$. En el cuadro No 3 damos los datos de mortalidad por tuberculosis en diferentes edades.

Respecto del sexo no se observa diferencias importántes en los 478 casos afectos de tuberculosis, pues corresponden al masculino $242(50.62 \%)$ y 236 a) femenino $(49.37 \%)$.

\section{b) Causa de muterte de los fallectios por entermedad no taberculoss.}

La causa de muerte de los 107 casos que no fallecieron por tuberculosis, es ta siguiente:

Toxemia ..

Meninglts .. . . . . . . . . . . . . . .. 10 "

purulenta (no tipificada) 4 casas

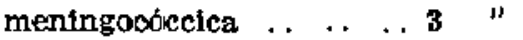

neumocócclca ....... 3 ,

Neumonía $\mathrm{y}$ bronconeumonía .. .. 10 "

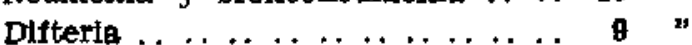

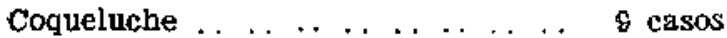

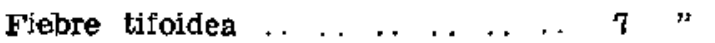

septicemia y septicopiohemia ... . 7 "

Fnterocolitis necrótico-fibrinosa .. 5 "

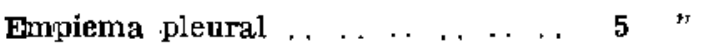

Peritonitis purulenta ... . . . . . . . 5 5 "

Reumatismo cardiaco .......... 4 "

Leucemia . . . .

Glomérulo-nefritis aguda y crónica 3 "

Laringttis pseudomembranosa . . . 2 " "

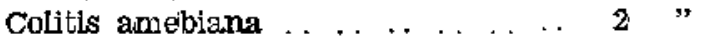

$\begin{array}{llllllllllll}\text { Caquexia } & . & \ldots & \ldots & \ldots & \ldots & \ldots & . & \ldots & 2 & & \end{array}$

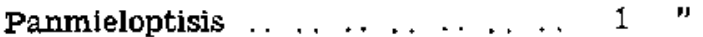

Tumor del cerebelo . .

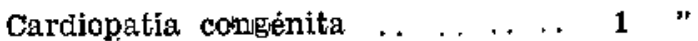

Cicactrices del miocardio $\ldots . . .6 .41$ "

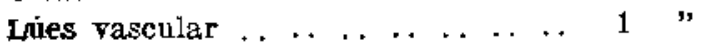

Hemorragla cerebral ...

II

\section{a) Complejo primario.}

En los 478 casos de niños con tuberculosis, encontramos el complejo primario como tal, o reducido sólo a uno de sus componentes en 462 casos, estando ausente en los otros 16 (cuadro $\left.N^{\circ} 4\right)$.

En resumen, tenemos:

Infección primaria pulmonar 456 casos $(98.70 \%)$

Infección primaria intestinal

$4 "(0.86 \%)$

Infección primaria de la cara

$1 *(0.21 \%)$

Infecclón primaria amlgdaliana

$1 "(0.21 \%)$

\section{b) Chancro primario pulmonar.}

Como queda dicho más arriba, en 277 casos se encontró el chancro primario pulmonar. Debido a la existencia en algunos do estos casos, de chancros múltiples, el número real de chancros ascendió a 296. La distribución de los chancros en los diferentes lóbulos pulmonares se encuentra en el cuadro $N^{\circ} 5$. 


\section{Guadro N:4 \\ Hallazgos del complejo primario en 478 casos con tubercułosis.}

Infección primaria pulmonar

Complejo primario pulmonar

Chancro puimonar solo

Adenopatía tráq.-br. sola

Isfección primaria extrapulmonar

Complejo primario intestinal

Adenopatia mesentérica sola

Adenopatía submaxilar y cervical derecha

Adenopatia preauricular y submaxilar dei echa

Total de infecciones primarias

Ausencia de complejo primario

Total
$456 \operatorname{cas} 0 \mathrm{~s}(98.70 \%)$

268 casos

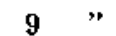

$179 "$

6 casos ( $1.29 \%)$
462 casos

16. casos

478 casos

Según el cuadro $N^{9} 5$, puede observarse qut el reparto do los chancros favorece por un margen importaute

\section{Cuadro N" 5}

Distribución de los chancros en ambos pulmones.

\begin{tabular}{lrllll}
\hline & P. D. & \multicolumn{1}{c}{$\%$} & P.I. & $\%$ \\
\hline Lóbulo sup. & 77 & 26 & & 59 & 19.33 \\
Lóbulo inf. & 66 & 22.29 & 59 & 19.33 \\
Lóbulo $1 / 2$ & 35 & 11.82 & & \\
& -178 & 60.13 & -118 & 39.80
\end{tabular}

al pulmón derecho $(60.13 \%$ contra $39.80 \%$ ) y que respecto de los lóbulos, en orden de frecuencia, son afectados Ios lóbulos superior derecho e inferior derecho, siguiendo en tercer lugar, en idéntica proporción, los lóbulos pulmonares izquierdos, y en último lugar, el lókulo medio derecho. Estos porcentajes se encuentran en general de acuerdo con lo que autores extranjoros indican en ste publicaciones. En priner lugar. Ja opirión es unánime en el sentido que el pulmón derecho es más frecuentemente afectado que el izquierdo, lo cual se atribuye a su mayor volumen, ya que genuralmente consta de 3 lóbulos. Res- pecto de la repartición de la infección primatia en los diferentes lóbulos, las cetadísticas dan resultados un tanto diferentes. Transcribimos a continuación (cuadro $\mathrm{N}^{\circ}$ 6) los porcentajes que diversos autores han encontrado sobre este aspecto y los comparamos con los muertros (citados por Simon y Redeker ${ }^{3}$ y W(ld $\left.{ }^{2}\right)$.

\section{Cuadro N 6}

Distribucion de las chancros en los diversos lóbulos en comparación con otros autores.

\begin{tabular}{|c|c|c|c|c|c|}
\hline \multirow{2}{*}{ Ghon Wintermitz } & \multicolumn{3}{|c|}{$\begin{array}{c}\begin{array}{c}\text { Pulmón } \\
\text { derecho }\end{array} \\
\begin{array}{lll}\text { L. s. } & \text { L. I } & \text { L. } 1 / 2\end{array}\end{array}$} & \multicolumn{2}{|c|}{$\begin{array}{l}\text { Pulmón } \\
\text { izquierdo } \\
\text { I. S. L. I. }\end{array}$} \\
\hline & 29.9 & 18.7 & 7.9 & 17 & .18 .3 \\
\hline Hesse & 28 & 18.6 & 10.1 & 17 & 26.4 \\
\hline Puhl & 32.1 & 17.1 & 10.7 & 28.5 & 11.6 \\
\hline Lange & 24.1 & 22.9 & 6.5 & 25.3 & 21.2 \\
\hline Blumenberg & 33.5 & 18.1 & $11: 6$ & 22.3 & 14.5 \\
\hline Weld & 41.4 & 27.6 & 6.9 & 13.7 & 10.4 \\
\hline Nosotros & 28 & 22.2 & 11.8 & 19.3 & 19.3 \\
\hline
\end{tabular}

Según Engel ${ }^{s}$, la distribución de los complejos primarios no se debe a la casualidad, sinn que asientan de preferencia en aquellas porciones pulmonares que están en comunicación con el exterior por un camino bronquial más cor- 
to $y$ en territorios en que está favorecido el andamiento de los bacilos por menor velocidad de la corriente aérea y lentitud de la circulación linfática. Este autor expliea así la preponderancia numérica de los complejos en las porciones posteriores del pulmón, región peri-hiliar $\mathrm{y}$ vértices.

En nuestro material encontramos chancros miúltiples en 12 casos, 10 de los cuales eran dobles, uno cuádruple y otro quíntuple. Enl 7 de los casos con chancro doble, éstos se encontraban en un nismo lóbulo y en los 5 canos restantes asentaban en lóbulos de ambos pulmones. EI porcentaje con que los autores consultados encuentran chancros múltiples, es muy variable: Ghon $y$ Wintermitz dan una cifra de $6.2 \%$ : Lange, $7.1 \%$; Hartmann, $8.3 \%$; Ickert, $9.4 \%$ y Puhl, $13 \%$. Para nosotros, la frecuencia de chancros múltiples es menot que los citados, pues alcanza sólo al $4.3 \%$. Porcentajes mucho más altos de chancros múltiples son dados por autores que han investigado estas lesiones mediante exámenes radiológicos de enfermos, pero como es sabido, este método da lugar a errores, ya que pueden diagnosticarse focos de diseminación linfática, sanguínea $\mathrm{O}$ canalicular como chancros múltiples. En la investigación radiológica, Simón ha encontrado chancros múltiples en ol $11.1 \%$, Ballin en el $15.9 \%$ y Muller y Klikmaun ell el $22 \%$. Respecto de la frecuencia de chancros dobles ell material di necropsia, Ghou los encuentra en el $8 \%$, en al $3 \%$ triples y un el $2 \%$ cuádruples. Blacklock da las cifras 7,3 y $1 \%$ de chancros dobles, triples y cuálruples, respectivamente. Nuestros resultados son de $3.58 \%$ de chimcros dobles y $0.35 \%$ de chancros cuádruples y quíntuples.

\section{c) Ausencia de una parte del complejo primario.}

Entre los 277 casos de infección priinaria pulmonar, a que más arriba nos bemos referido, en 9 casos no se encontró la parte ganglionar correspondiente. Dacimos "no se encontró", ya que no podemos asegurar con absoluta certeza que no existiera, porque en sn búsqueda sólo utilizamos el examen macroscópico de los ganglios regionales. Creenos, sin embargo. que las posibilidades de error no puedel alcanzar al total de los $9 \mathrm{ca}$ sos, por las consideraciones que más adelante expresaremos. De este grupo de niños (cuadro $\mathrm{N}^{\circ}$ 7) la edad oscilaba entro 1 mes 26 días y 11 años, y su mayor número (7 casos) entre 5 y 11 años. En 5 casos no se encontraron en

Cuadro N: 7

Auseneia de la parte ganglionar del complejo primario ( 9 casos).

Estado físico chaneros.

\begin{tabular}{|c|c|c|c|c|c|}
\hline Edad & $\begin{array}{l}\text { No de } \\
\text { casos }\end{array}$ & Caseoso & Cretáceo & Calcificado & Causa de muerte \\
\hline I. mes 26 dias & 1 & sí & 一 & - & Cardiopatía congénita \\
\hline 1 año 3 meses & 1 & sí & - & 一 & Toxemia \\
\hline 5 años & 1 & - & si & - & $\begin{array}{l}\text { Men. the. y mil. gen. } \\
\text { (Estreptomic.l. }\end{array}$ \\
\hline 8 años & 2 & sí & - & sí & $\begin{array}{l}\text { 1) Osteitis tbe multip. Ca- } \\
\text { quexia. } \\
\text { 2) F. tifoidea. }\end{array}$ \\
\hline 9 años & 1 & - & $\longrightarrow$ & si & $\begin{array}{l}\text { M'en. tbe. y mil. gen. } \\
\text { Tbe. genital. }\end{array}$ \\
\hline 10 años & 2 & - & - - & sí(2) & $\begin{array}{l}\text { 1) F. tifoídea. } \\
21 \text { Men. tbc. } \\
\text { (Estreptomic.). }\end{array}$ \\
\hline 11 años & 1 & 一 & sí & - & Iúks vascular. \\
\hline
\end{tabular}


el resto del organismo otras lesiones tuberculosas fuera del chancro pulmonar y la causa de muerte fué, en consecuencia, ajena a la tuberculosis. En 3 casos hubo diseminaciones hematógenas (meningitis y miliar generalizada), que fueron la causa del fallecimiento, uno de los cuales (niño de 9 años) tenía un foco antigno extrapulmonar (tuberculosis genital), mientras que en los otros 2 se encontró el chancro como única fuente de diseminación. Finalmente, 1 caso murió en caquexia por osteítis tuberculosas múltiples.

Debemos agregar que en 2 casos, como está señalado en el cuadro, se hizo tratarniento intenso con estreptomicina, a la cual podría imputarse una rápida y total regresión de la adenopatía, que no permitió su hallazgo macroscópico. Aunque nuestra experiencia histológica no es grande en los resultados de esta terapéutica, podemos adelantar que en tales casos las lesiones tuberculosas por nosotros encontradas, pueden alcanzar a la cicatrización por fibrosis y hasta calcificación, lo cual es evidente aún en gránulos miliares, que en tal estado son apreciables al simple examen macroscópico. Igual experiencia manifiestan Clarke y Silverman ${ }^{6}$ y Mahon ${ }^{7}$ en recientes publicaciones al respecto. Tratándose de ganglios o chancros en esta etapa de curación, lógicamente, tampoco es fácil que puedan pasar inadvertidas, más aún si el chancro pulmonar correspondiente, como en uno de los casos a que nos referimos, estaba todavía en estado de cretificación. Por esto, creemos que difícilmente la estreptomicinoterapia llegue, en su poder curativo, a hacer desaparecer totalmente, hasta las secuelas fibrosas o cálcicas de las lesiones tnberculosas ganglionares y si tal cosa sucediera, $\operatorname{los} 7$ casos restantes, no tratados con dicho medicamento, sirven para los efecto's del tema que comentamos.

Indudablemente llama la atención el no hallazgo de la parte ganglionar del complejo primario en los 9 casos a que nos estamos refiriendo, habiéndolos bascado con intención en la región corres- pondiente de los ganglios tráqueo-bronquiales. Especialmente esto parece extraño si recordamos que la porción ganglionar del complejo primario evoluciona más lentamente a la curación que la respectiva parte parenquimatosa, en tal forma, que es frecuente observar ganglios caseosos o cretáceos, correspondientes a chancros cretáceos a calcificados. respectivamente. Vale la pena añadir que en uno de nuestros casos (niña de 8 años) había chancro primario doble en el lóbulo inferior del pulmón izquierdo, en estado caseoso. En el caso del niño más joven ( 1 mes 26 días) y que presentaba un chancro tuberculoso caseificado indubitable, confirmado por exámenes microscópicos (aun coloración específica del bacilo de Koch intensamente positiva en cortes histológicos del chancro), podríamos suponer por la edad del niño y el estado físico del chancro, que la temprana muerte del enfermo hubiera impedido la propagación de la infección pulmonar a los ganglios satélites. Sin embargo, tal explicación no cabe. pues Ghon ${ }^{8}$ y Román ${ }^{b}$ han demostrado que los ganglios regionales comprometen antes que el chancro se caseifique.

En la literatura consultada se registran varios casos, en los cuales se ha comprobado la ausencia de la parte ganglionar del complejo primario. Terplan ${ }^{1}$, que hace una revisión de los casos publicados hasta 1940 , refiere 4 casos pertenecientes a Ghon (2 casos), Kudlich (1 caso) y Blumenberg (1 caso), de 7, 5, 13 años y 3 meses, respectivamente, en los cuales, aun con el examen microscópico seriado de los ganglios regionales practicados en 2 de ellos, no enoontraron lesiones tuberculosas activas o residuales. Agrega Terplan 5 casos propios ( 2 de ellos con 13 y 24 focos primarios, respectivamente), en los cuales la investigación macro y microscópica de los ganglios satélites del chancro fué negativa. En todos los casos citados, además, no se comprobaron diseminaciones tuberculosas intrapulmonares ni de otros órganos. Estos hechos 
los interpreta Terplan como "una feliz resistencia tisular", ya que la lesión primaria queda réducida al parénquima pulmonar. Sin embargo, la limitación excepcional del proceso no impide, como lo demuestran 4 de los 9 casos nuestros, a que por vía hematógena el chancro dé lugar a diseminaciones extrapulmonares de tal cuantía y gravedad como aquellos otros, que son la mayoría y que se acompañan de adenopatía satélite.

Estas observaciones tienen también importancia en el terreno de la patología general de la tuberculosis, pues modifican el criterio mantenido durante tantos años y que se sintetizó en la llamada "ley de Parrot", que establecía que en la lesión primaria, los ganglios que drenan la zona de infección están siempre comprometidos. Los hechos expuestos prueban fehacientemente que hay excepciones a la mencionada ley.

\section{d) Infección primaria extrapalmonar.}

Como ya dejamos consignado, en nuestro material encontramos 6 casos en que la infección primaria tuberculosa se hizo fuera del pulmón, lo que representa una cifra de $1.29 \%$. De estas 6 casos, 4 corresponden a primo-infección intestinal, 1 a primo-infección amigdaliana y 1 a primo-infección de la cara. En un reciente trabajo de Peña Cereceda y Barilari ${ }^{10}$, estos colegas, en una revisión de 6,000 observaciones clínicas de enfermos tnberculosos, daban cuenta de la presencia de 35 casos de chancro primario extrapulmonar lo que significa una incidencia de $0.58 \%, 21$ de los cuales eran cutáneo-mucosos (piel de las piernas y cara, conjuntiva ocular y genitales) y 14 de la cavidad bucal (especialmente de las amigdalas). En todos sus casos, los autores dejan cons. tancia de la no participación del pulmón en la evolución posterior de la infección primaria, durante uni lapso de observación variable entre 2 y 7 años, así como también de la ausencia de diseminaciones tuberculosas a partir del chancro primario. En el aspecto clínico, esto ha- blaría en favor de la benignidad de la infección primaria tuberculosa extrapulmonar.

Nuestros 4 casos de primo-infección intestinal significa un valor $0.86 \%$ respecto de la localización de la infección primaría encontraáa en ruestro material. Esta cifra está cercana a la obtenida por otros autores en material necrópsico. Así, por ejemplo, Pirquet ${ }^{23}$ da el $0.43 \%$ de infección primaria intestinal, Ghon, Hansemian, Baginsky y Albrecht (citados por Simón y Redeker ${ }^{3}$ ) dan el $1 \%$, Puhl da el $1.8 \%$, Steben el $5 \%$ y Terplan el $7 \%$. Todos los autores están de acuerdo en que, debiéndose la primo-infección intestinal al bacilo bovino, ésta es más frecuente en aquellos países o lugates donde el consumo de leche, especialmente cruda, es mayor. Tal cosa sucede en Inglaterra, Escocia, Países Bàjos, algunas regiones de Alemania, EE. UU., etc, En Kiel, por ejemplo, Heller ${ }^{11}$ ha encontrado una proporción del $21 \%$ de focos entéricos bovinos.

Brevemente resumiremos los datos anatómicos de mayor interés de los 4 casos de infección primaria intestinal.

Caso 1. - Niño de 8 años (Necropsia 186 / 47), en el cual se encontró un ganglio mesentérico cretificado y sin otras alteraciones tuberculosas en el resto del organismo. Su causa de muerte fué peritonitís purulenta generalizada, secundaria a perforación de la vesícula biliar por gangrena.

Caso 2. $\rightarrow$ Niña de 2 años (Necropsia $\mathbb{N}^{\circ}$ $307 / 47$ ) presentaba una úlcera tuberculosa del íleon a $5 \mathrm{~cm}$ de la vá]vula ileo-cecal, del tamaño de una moneda de reinte centaros y rartos ganglios íleo-cecales totalmente caseosos. No se encontraron lesiones tuberculosas en otros órganos y su falleclmiento se debló a laringitis pseudomembranosa.

Caso 3. - Ntña de 14 años (Necropsla No 155/49) presentaiba un ganglio mesentérico calcificado, In el lóbulo supertor lzquierdo se encontró, además, una caverna de tipo crónico del tamaño de una nuez, que consideramos de retnfección y la causa de la muerte fué tuberculosis acinosa productiva de arnbos pulmones, de regular intensidad. 
Caso 4. - Niño de 8 meses (Necropsia N $177 / 49$ ), con intensa adenoipatía tuberculosa caseosa de los ganglios mesentéricos, peripancreáticos, de la curvadura menor del estómago $\mathrm{y}$ del bilio hepático, ausencla de ganglios tuberculosos en el tórax y muerte por meningitis tuberculosa y miliar generalizada.

En resumen, $\operatorname{los} 4$ casos presentaban e]ementos anatómicos indubitables de infección primaria intestinal, 1 con la parte parenquimatoma y ganglionar del complejo primario y los otros 3 con solo la adenopatía mesentérica correspondiente. En el caso 4 la adenopatía tuberculosa del mesenterio se propagó a los ganglios vecinos (peri-pancreáticos, curvadura menor del estómago y del hilio hepático), encontrándose todos éstos totalunente caseificados. En todos los casos no se encontraron lesiones tuberculosas pulmonares ni de los ganglios tráqueobronquiales que pudieran imputarse a infección primaria y sólo en uno de ellos (niña de 14 años) coexistía una caverna tuberculosa crónica de un lóbulo pulmonar que consideramos de reinfección.

Respecto de otras localizaciones extrapulmonares de la primo-infección, encontramos 2 casos, uno de ellos (niño de 12 años, Necropsia $N^{9} 472 / 44$ ) con adonopatía tubetculosa caseosa de la región submaxilar derecha y cervical del mismo lado, ganglios que por ser tributarios de la amígdala derecha consideramos que éste ha sido el órgano afectado por la infección primaria, aunque macroscópicamente no revelaba alteraciones, y el segundo caso (niña de 6 años, Nectopsia $N^{\circ}$ 291/45) con adenopatía tuberculosa caseosa de la región preauricular derecha y submaxilar del mismo lado, ganglios que por ser afluentes de la cara (incluso ojo y nariz), suponemos que en esta región se produjo el chancro primario, aunque al tiempo de su muerte no se encontraron lesiones a ese nivel. En estes dos casos tampoco se comprobaron lesiones tuberculosas de los pulmones ni de los ganglios tráqueobronquiales del tipo de la lesión pri- maria, La causa de muerte en ambos niños fué meningitis tuberculosa y miliar generalizada, cuyo punto de partida $n o$ pudo ser otro sino la adenopatía del complejo primario. Desgraciadamente, la historia elínica de ambos casos no anotaba nimgún antecedente que pudiera servir para certificar la ubicación del chancho primario en los sitios a que por los hallazgos anatómicos de la adenopatía les hemos atribuido.

Como decíanios al empezar a hablar de los complejos extrapulmonares, Peña Cereceda y Barilari observaron que en sus casos no hubo diseminaciones e igual experiencía manifiesta. Terplan respecto de aquellos casos con primo-infección intestinal, lo cual hablaría en favor de la benignidad clínica de estos tipos de infección primaria. Sin embargo, nuestra experiencia es diferente, ya que de los 6 casos de complejo extrapulmonar, 3 fallecieron por diseminación hematógena (meningitis tuberculosa y miliar generalizada), cuyo punto de partida no pudo ser sino la adenopatía caseosa del complejo primario, puesto que no había otros focos tuberculosos que pudieran generar tales complicaciones.

\section{e) Ausencia de complejo primario.}

En 16 casos (3.34\%) hubo ausencia completa del complejo primario. En casi todos estos casos, a excepción de uno do 10 meses, la edad oscilaba entre 3 y 16 años. El de 10 meses murió de meringitis tuberculosa y miliar generalizada y sin otras lesiones tuberculosas en sus órganos linfoídeos y parenquimatosos, no habiendo sido tratado tampoco con estreptomicina. En 3 casos (niños de 10,11 y 16 años) se encontró una tuberculosis cavitaria múltiple de los pnlmones, muy probablemente de reinfección, cuyos ganglios tráqueo-bronquiales mostraban o leves signos de tuberculosis miliar productiva o ninguna lesión tuberculosa macroscópica. En otros 11 casos, cuya edad oscilaba entre 3 y 11 años, se encontró igual que en el 
niño de 10 meses, lesiones de meningitis tuberculosa y miliar generalizada. En sólo 3 de éstos se observó una tuberculosis orgánica como fuente visible de la diseminación hematógena: Mal de Pott en 2 casos y artritis de la rodilla en 1 . De estos 12 casos, 7 fueron tratados con estreptomirina. Finalmente, en 1 caso, sólo se encontró artritis tuberculosa de una cadera y la causa de muerte fué una leucemia.

La ausencia del complejo primario es en realidad de difícil explicación. Podría suponerse, para los niños mayores, una tan avanzada involución de dichas lesiones, que no hiciera posible su comprobación macroscópica, pero esto no cabría para aquellos casos de pocos meses o años, en los que se trate de primoinfección pulmonar o extrapulmonar, no es corriente la desaparición completa o casi completa, no sólo de la parte parenquimatosa, que en caso de primo-infección extrapulmonar es de involución rápida, sino especialmente de su porción ganglionar, que en general va a la curación en forma lenta $\mathrm{y}$ gradual. En aquellos niños con tuberculosis cavitaria pulmonar de reinfección ( 3 casos) podría suponerse que el chancro quedó incluído en las formaciones cavernosas y se reblandeció con ellas, pero tampoco nos explica la desaparición macrostópica de la adenopatía tráqueo-bronquial correspondiente. Es también motivo de interrogación el punto de partida que tuvienon las generalizaciones hematógenas en 9 de estos casos, ya que en los otros 3 se encontró un foco de tuberculosis orgánica. Es evidente que tal foco debió existir, pero de cuantía tan peque$\bar{n}$ a, que pasó totalmente desapercibido al examen macroscópico. Podria tal vez tratarse del llamado "tubérculo de la intima", según el concepto de Benda ${ }^{12}$ y Schmorl ${ }^{13}$, lesión que, como se sabe, es muy difícil de encontrar si no se investiga prolijamente en la intima $d_{0}$ las venas pulmonares, espermáticas, de las cápsulas suprarrenales, de los huesos, eti., búsqueda que en nuestro material, porr desgracia, no se realizó.
$\mathrm{III}$

Estado físico del chancro pulmonar.

Como queda dicho más arriba, el chancro fué encontrado en 277 casos, pero por la existencia de chancros múltiples, su número alcanzó a 296. Entre los chancros incluímos las cavernas primarias, ya que derivan directamente de aquéllos. El estado físico on que se en-

\section{Cuadro $\mathrm{N}^{\circ} 8$}

Estado físico de Ios chancros pulmonares (296).

\begin{tabular}{lrc}
\hline & Ni chancros & Porcentaje \\
\hline Casificados & 194 & 85.54 \\
Cretificados & 62 & 17.56 \\
Calcifjcados & 23 & 7.77 \\
Cavernas primarias & 27 & 9.12
\end{tabular}

contraton los chancros los resumimos en el cuadro $N^{\circ} 8$. Como puede observarse, las cantidades son sensiblemente decrecientes desde los chancros caseifica-

Cuadro $\mathbf{N}^{* 9}$
Porcentaje de los diversos tipos de chancros pulmonares según la edad de los easos respectivas:

\begin{tabular}{rrrrc}
\hline Edades & $\begin{array}{c}\text { Caseo- } \\
\text { sos }\end{array}$ & $\begin{array}{c}\text { Cretá- } \\
\text { ceos }\end{array}$ & $\begin{array}{c}\text { CaJci- } \\
\text { ticados }\end{array}$ & $\begin{array}{c}\text { Caverna } \\
\text { prim. }\end{array}$ \\
\hline 0-12 meses & $\mathbf{3 2 . 9 8}$ & 9.6 & - & 40.74 \\
12-24 meses & $\mathbf{2 6 . 2 8}$ & $\mathbf{2 1 . 1 5}$ & - & $\mathbf{2 2 . 2 2}$ \\
2- 3 años & 12.37 & 9.61 & 13.04 & 14.81 \\
3- 4 años & 6.70 & 5.76 & 8.69 & 7.40 \\
$4-6$ años & $\mathbf{7 . 2 1}$ & $\mathbf{2 6 . 9 2}$ & - & - \\
$6-8$ años & 5.15 & 19.23 & 17.39 & $\mathbf{3 . 7 0}$ \\
8-10 años & 3.60 & - & 30.43 & 11.11 \\
$10-12$ años & 5.15 & 5.76 & 26.08 & - \\
$12-14$ años & 0.51 & 1.92 & - & - \\
$14-16$ años & - & - & 4.34 & -
\end{tabular}

rios a los cretificados y calcificados. Es de interés observar estos mismos porcentajes según la edad de los casos (cuadro No 9). Como era de esperar, alrededor del $70 \%$ de los chancros caseificados se observan en $\operatorname{los} 3$ primeros años de la vida, ya que en estas edades 
se produce en tulestro medio la mayor cantidad de primo-infecciones, proporción que disminuye gradualmente hacia las edades superiores. Respecto del chaucro cretáceo, llama la atención su presencia en un $10 \%$ en el primer año de la vida y en un $21 \%$ en el segundo año, o sea, el $30 \%$ más a menos de los chancros cretificados encuentran en los 2 primeros años de la vida, lo cual da una idea de la precocidad de la infección primaria en nuestro material, ya que debemos suponer una evolución de varios meses para que el chancro alcance este estado físico. Debemos hacer presente que en ninguno de estos casos con chancro cretifícado se usó estreptomicina. En cuanto a los chancros calcificados, éstos aparecen ya en una cantidad importante entre los 2 y 3 años (13\%), lo cual confirma los comentarios anteriores respecto de la infección primatia precoz en nuestro medio. Finalmente, las cavernas primarias tienen un mayor porcentaje en el primer año de la vida $(41 \%)$, disminuyendo progresivamente hacia las edades superiores, lo que nos hace suponer la gravedad que significa para el recién nacido - lactante la infección tuberculosa, hecho que también debemos relacionarlo con una infección masiva de éste, que rebalsa su capacidad de resistencia para limitar el proceso. Respecto de la ubicación de las cavernas primarias y del se$\mathrm{xo}$ correspondiente a los casos en referencia, vet cuadro $N^{9} 10$, que no necesita comentarios.

Entre los 194 chancros caseificados se encuentran 16 chancros reblandecidos $(8.24 \%)$ y $22(11.34 \%)$ con excavación central incipiente (cuadro $\mathrm{N}^{\circ} 11$ ). $\mathrm{Su}$ mayor porcentaje se observa en los primeros 2 años de la vida (81 y $63 \%$, respectivamente), muy probablemente en relación con una infección tuberculosa con gran cantidad de bacilos de Koch o con defensas orgánicas muy débiles, que impiden una limitación adecuada del pnoceso o ambas causas a la vez. La evolución del chancro al reblandecimiento y excavación, significan eviden-
Cuadro N 10

Ubicación de las cavernas primarias.

$\begin{array}{lllr}\text { Pulmón derecho: } & \text { lóbulo superior } & 14 \\ & " & \text { medio } & 0 \\ \text { Pulmón izquierdo: } & " & \text { inferior } & 6 \\ & " & \text { superior } & 6 \\ & & & 1 \\ & & \text { Total } & 27\end{array}$

Sexo de los casos con cavernas primarias.

$\begin{array}{rr}\text { Femenino } & 14 \\ \text { Masculino } & 13 \\ & - \\ \text { Total } & 27\end{array}$

Cuadro N 11

Chancros caseificados reblandectdos y excavados en relación con la edad.

\begin{tabular}{|c|c|c|}
\hline Edades & $\begin{array}{c}\text { Chancros } \\
\text { reblandectios }\end{array}$ & $\begin{array}{l}\text { Chancros } \\
\text { excavados }\end{array}$ \\
\hline $0-12$ meses & $4 \int_{81 \% 5 \%}$ & ${ }^{7} \int_{6363 \%}$ \\
\hline 12-24 meses & 9 & 7 ] \\
\hline 2- 3 años & 一 & 3 \\
\hline 3- 4 años & 1 & 2 \\
\hline $4-6$ años & 1 & 2 \\
\hline 6-10 años & 1 & - \\
\hline 10-16 años & 一 & 1 \\
\hline Totales & 16 & 22 \\
\hline
\end{tabular}

Cuadro $\mathbf{N}^{\circ} 12$

Propagación intrabronquial en chancros caseosos y cavemas primarlas.

\begin{tabular}{lcc}
\hline & $\begin{array}{c}N^{\prime} \\
\text { chancros }\end{array}$ & $\begin{array}{c}\text { Porcentrie } \\
\text { de positiv. }\end{array}$ \\
\hline $\begin{array}{l}\text { Chancros caseosos } \\
\text { Chancros caseosos y re- } \\
\text { blandecidas }\end{array}$ & 156 & 21.15 \\
$\begin{array}{l}\text { Chancros caseosos exca- } \\
\text { vados }\end{array}$ & 16 & 6.25 \\
$\begin{array}{l}\text { Cavernas primaries } \\
\text { a }\end{array}$ & 22 & $\mathbf{4 5 . 4 5}$ \\
\end{tabular}


los peligros de generalización precoz, ya por vía hematógena a intracanalicucar (intrabronquial). En el cuadro $\mathrm{N}^{9} 12$ podemos observar el porcentaje de propagación intrabronquial en los diversos tipos de chancro y en las cavernas primarias. Allí se revela en forma elocuentte el alto porcentaje do esta complicación en los chancros caseosos excavados y en las cavernas primarias. Llama la atención la baja cifra con que participan en el proceso que comentamos, los chanchos caseosos reblandecidos, pese a que teóricamente judiéramos suponer una mayor proporción que los caseosos puros, los cuales lo realizan en un $21 \%$. Respecto de estos últimos tenemos que admitir que su acceso al lumen bronquial debe realizarse mediante la caseificación y reblandecimiento del mismo $y$ de la pared bronquial, proceso que en la mayoría de los casos sólo al examen microscópico es posible verificarlo. Sin embargo, juegan también importante papel en la propagación intrabronquial de la tuberculosis, las perforaciones groseras, macroscópicas, del chancro a un bronquio grueso 0 a alguna de sus ramificaciones y las nleeraiciones tuberculosas de la mucosa del árbol respiratorio superior (laringe, tráquea, grandes bronquios) y de la faringe (amígdalas), focos que en forma permanente y en intensidad variable bombardean los pul- mones con material bacilífero. En el caso de los chancros caseosos, en una oportunidad se encontró el chancro perforado a un bronquio, en otras dos ocasiones había úlceras múltiples de la mucosa del aparato respiratorio superior y en 6 casos, el chancro coexistía con perforación de un ganglio tráqueo-bronquial al lumen de un bronquio. También en los casos de chancros caseosos excavados, en 3 oportunidades había perforación de brouquios por ganglios, lo cual es un poderoso elemento que contribuye o determina la propagación tuberculosa intrabronquial. Finalmente, deseamos hacer notar la ausencia de la tuberculosis canalicular del pulmón en un porcentaje importante de los chan. chos caseificados y reblandecidos y aun en los excavados y cavernas primarias. Es más sorprendente este fenómeno en los dos últimos nombrados (54 y $40 \%$, respectivamente), ya que en ellos es evidente la invasión bacilífera al parénquima pulmonar. Su única explicación estaría en suponer eficientes medios de defersa local $y$ general (inmunidad) para oponerse a la proliferación destructiva de los gérmenes específicos o que el material caseoso no tenga contenido bacilífero virulento suficiente.

(Continuará). 